\title{
Integer least-squares theory for the GNSS compass
}

\author{
P. J. G. Teunissen
}

Received: 31 December 2009 / Accepted: 11 March 2010 / Published online: 2 April 2010

(C) The Author(s) 2010. This article is published with open access at Springerlink.com

\begin{abstract}
Global navigation satellite system (GNSS) carrier phase integer ambiguity resolution is the key to highprecision positioning and attitude determination. In this contribution, we develop new integer least-squares (ILS) theory for the GNSS compass model, together with efficient integer search strategies. It extends current unconstrained ILS theory to the nonlinearly constrained case, an extension that is particularly suited for precise attitude determination. As opposed to current practice, our method does proper justice to the a priori given information. The nonlinear baseline constraint is fully integrated into the ambiguity objective function, thereby receiving a proper weighting in its minimization and providing guidance for the integer search. Different search strategies are developed to compute exact and approximate solutions of the nonlinear constrained ILS problem. Their applicability depends on the strength of the GNSS model and on the length of the baseline. Two of the presented search strategies, a global and a local one, are based on the use of an ellipsoidal search space. This has the advantage that standard methods can be applied. The global ellipsoidal search strategy is applicable to GNSS models of sufficient strength, while the local ellipsoidal search strategy is applicable to models for which the baseline lengths are not too small. We also develop search strategies for the most challenging case, namely when the curvature of the non-ellipsoidal ambiguity search space needs to be taken into account. Two
\end{abstract}

P. J. G. Teunissen

Department of Spatial Sciences, Curtin University of Technology, Perth, Australia

e-mail: p.teunissen@curtin.edu.au

P. J. G. Teunissen ( $\varangle)$

Delft Institute for Earth Observation and Space Systems (DEOS),

Delft University of Technology, Delft, The Netherlands

e-mail: p.j.g.teunissen@tudelft.nl such strategies are presented, an approximate one and a rigorous, somewhat more complex, one. The approximate one is applicable when the fixed baseline variance matrix is close to diagonal. Both methods make use of a search and shrink strategy. The rigorous solution is efficiently obtained by means of a search and shrink strategy that uses nonquadratic, but easy-to-evaluate, bounding functions of the ambiguity objective function. The theory presented is generally valid and it is not restricted to any particular GNSS or combination of GNSSs. Its general applicability also applies to the measurement scenarios (e.g. single-epoch vs. multiepoch, or single-frequency vs. multi-frequency). In particular it is applicable to the most challenging case of unaided, single frequency, single epoch GNSS attitude determination. The success rate performance of the different methods is also illustrated.

Keywords GNSS - Attitude determination . Integer ambiguity resolution - Constrained integer least squares

\section{Introduction}

Global navigation satellite system (GNSS) integer ambiguity resolution (IAR) is the process of resolving the unknown cycle ambiguities of the carrier phase data as integers. The sole purpose of ambiguity resolution is to use the integer ambiguity constraints as a means of improving significantly on the precision of the remaining model parameters. Once this has been done successfully, the carrier phase data will act as very precise pseudo range data, thus making very precise positioning and navigation possible. IAR applies to a great variety of current and future GNSS models (e.g., GPS, modernized GPS, Galileo), with a wide range of applications, 
such as those in surveying, navigation, geodesy, and geophysics. These models may differ greatly in complexity and diversity. They range from single-baseline models used for kinematic positioning to multibaseline models used as a tool for studying geodynamic phenomena. An overview of these models can be found in textbooks, such as Parkinson and Spilker (1996), Strang and Borre (1997), Teunissen and Kleusberg (1998), Hofmann-Wellenhof et al., Leick (2003), Misra and Enge (2006), Hofmann-Wellenhof et al. (2008).

In this contribution, we consider the problem of precise GNSS attitude determination. GNSS attitude determination is a rich field of current studies, with a wide variety of challenging (terrestrial, sea, air, and space) applications. The earliest methods of attitude IAR are the so-called motion-based methods (see e.g. Cohen and Parkinson 1992; Chun and Park 1995; Tu et al. 1996; Crassidis et al. 1999). These methods take advantage of the change in receiver-satellite geometry that is induced by the platform's motion. They are not applicable however on an epoch-by-epoch basis, as the presence of motion is needed per se.

Another class of methods is the class of search-based methods (see e.g. Knight 1994; Park et al. 1996; Juang and Huang 1997). These methods are not necessarily dependent on motion and can therefore be used instantaneously in principle. They differ in the search-domain used and in the objective function to be optimized. The method of Knight (1994), for instance, searches for the optimal relative antenna positions. It requires some initial knowledge of the platform's attitude, in the absence of which the method is reported to become problematic (Gomez and Lammers 2004). Also in the method of Park et al. (1996), relative antenna positions are searched. This method divides, just like some of the older baseline ambiguity resolution methods, the $n$-dimensional integer ambiguity vector into a three-dimensional so-called independent part and an $(n-3)$-dimensional dependent part (see e.g. Hatch 1990). The independent part is then used in the baseline search.

In Juang and Huang (1997), the ambiguity function method is used. Counselman and Gourevitch (1981) proposed this principle, Mader (1990) and Remondi (1990) further investigated it for surveying applications. The method has the attractive property of being invariant for integer cycle slips. However, it has a complex multi-peak search space, based on a multivariate summation of trigonometric functions. Therefore, its integer search still poses a major challenge (see e.g. Li et al. 2004; Wang et al. 2007). Hence, just as in case of surveying, the ambiguity function method for attitude determination is not yet widely used.

More recent attitude determination methods, search in the ambiguity domain. Several of them make use of the LAMBDA method (see e.g. Furuno 2003; Lin et al. 2004; Monikes et al. 2005; Kuylen et al. 2006; Hauschild et al. 2008; Wang et al. 2009a). This method is known to be effi- cient and known to maximize the ambiguity success rate (Teunissen 1995, 1999). However, since the method has been developed for unconstrained and/or linearly constrained GNSS models, it is not necessarily optimal for the nonlinear GNSS attitude model. The nonlinearity stems from the a priori given baseline length. Many of the existing methods make use of this additional information, by checking whether or not the candidate baselines satisfy the given baseline length. Although this usage of the given baseline length indeed improves ambiguity resolution, such methods are still ad hoc as this validity check does not do full justice to the given information.

This observation has motivated the present author to develop a new and rigorous attitude IAR method based on the integer least-squares principle as optimality criterium. In this contribution, we develop a nonlinear constrained integer least-squares theory, together with integer search strategies, for an optimal and efficient estimation of the integer ambiguities. As opposed to current practice, our method does proper justice to the a priori given information. The nonlinear baseline constraint is fully integrated into the ambiguity objective function, thereby receiving a proper weighting in its minimization and providing guidance for the integer search. The theory presented is generally valid and it is not restricted to any particular GNSS or combination of GNSSs. Its general applicability also applies to the measurement scenarios (e.g. single-epoch vs. multi-epoch, or single-frequency vs. multifrequency).

This contribution is organized as follows. In Sect. 2, we briefly review IAR for the standard GNSS model. When considering IAR for attitude determination, one should keep in mind that the difficulty of computing and estimating the integer ambiguities depends very much on the strength of the underlying model. In case such models have sufficient strength, standard IAR methods can be used directly for precise attitude determination. This will be the case, for instance, when GNSS is externally aided with additional sensors (e.g. inertial measurement unit IMU). It will also be easier when multiple epochs and/or multiple frequencies are used. The challenge, however, is to be able to perform successful and efficient IAR for the unaided, single frequency, single epoch case. Since the standard version of these models lack the necessary strength, additional information in the form of given baseline lengths is often used in case of attitude determination. The incorporation of this constraint leads to the GNSS compass model, as discussed in Sect. 3. Although the use of baseline length information for IAR is not new, our model formulation and method of solution are new. We introduce our nonlinear constrained integer least-squares principle and derive the corresponding new ambiguity and baseline estimators. As a result, we obtain a new ambiguity objective function, that clearly reveals how the baseline length constraint and the integer ambiguity constraints are integrated 
in the estimation process. By comparison of ambiguity objective functions, we also show the difference between our estimators and the ones used in the literature.

Our ambiguity objective function is nonquadratic, which implies that its search space is nonellipsoidal. Straightforward application of the LAMBDA method is, therefore, not possible. This means that a new search strategy needs to be devised. However, if it is possible, in one way or the other, to make use of an ellipsoidal search space, one would be in the agreeable position that the standard LAMBDA method can be used again. In Sect. 4, two different ellipsoidal search strategies are presented; a global one and a local one. The global search strategy works for GNSS models of sufficient strength, while the local one works for GNSS models of which the baselines are sufficiently long. Our IAR derivations will be based on the weighted constrained integer least-squares principle. This has the advantage that our methods and analysis hold true for both the unconstrained and the hard constrained case.

In Sect. 5, we start by introducing a useful simplification of the nonellipsoidal search space. This results in another two search strategies, an approximate one and an exact one. Both strategies explicitly exploit the geometry of the nonellipsoidal search space and they both avoid the computational intensive evaluation of the ambiguity objective function during the search. The first one, being somewhat simpler than the second, solves the integer minimizer in an approximate sense, while the second strategy solves the integer minimizer in a global and exact sense. The advantage of the first strategy over Sect. 4 local ellipsoidal search is that its performance is not dependent on how long the baseline is. The second strategy is designed for the most challenging case, being unaided, single-epoch, single-frequency attitude IAR. Due to the complexity involved, particular attention is given to the size setting of the nonellipsoidal search space and to the search and shrink strategy employed. We also show the significant improvement in success rate performance that can be expected of this method when compared to the unconstrained case.

\section{The standard GNSS model}

\subsection{Heading and elevation}

In principle, all the GNSS baseline models can be cast in the following frame of linear(ized) observation equations,

$\mathrm{E}(y)=A a+B b, a \in \mathbb{Z}^{n}, b \in \mathbb{R}^{p}, \quad \mathrm{D}(y)=Q_{y y}$

where $y$ is the given GNSS data vector of order $m$, and $a$ and $b$ are the unknown parameter vectors of order $n$ and $p$ respectively. $\mathrm{E}(\cdot)$ and $\mathrm{D}(\cdot)$ denote the expectation and dispersion operators, respectively, and $A$ and $B$ are the given design matrices that link the data vector to the unknown parameters. Matrix $(A, B)$ is assumed to be of full rank. Matrix $A$ contains the carrier wavelengths and the geometry matrix $B$ contains the receiver-satellite unit line-of-sight vectors. The variance matrix of $y$ is given by the positive definite matrix $Q_{y y}$. The data vector $y$ will usually consist of the 'observed minus computed' single- or multi-frequency double- difference (DD) phase and/or pseudorange (code) observations. The entries of vector $a$ are then the DD carrier phase ambiguities, expressed in units of cycles rather than range. They are known to be integers, $a \in \mathbb{Z}^{n}$. The entries of the vector $b$ will consist of the remaining unknown parameters, such as baseline components (coordinates) and possibly atmospheric delay parameters (troposphere, ionosphere). They are known to be real-valued, $b \in \mathbb{R}^{p}$. Vectors $a$ and $b$ are referred to as the ambiguity vector and the baseline vector, respectively.

Since we consider the GNSS-compass application in the present contribution, we restrict attention to the case of satellite tracking with two near-by antennas. The short distance between the two antennas implies that we may neglect the (differential) atmospheric delays. Thus $p=3$ and $b=$ $\left(b_{1}, b_{2}, b_{3}\right)^{\mathrm{T}} \in \mathbb{R}^{3}$ consists then only of the three coordinates of the baseline vector between the two antennas. If the baseline vector is parametrized with respect to the local North-East-Up frame, the heading $H$ and elevation $E$ can be computed from the baseline coordinates $b_{1}, b_{2}$ and $b_{3}$ as

$H=\arctan \frac{b_{2}}{b_{1}}$ and $E=\arctan \frac{b_{3}}{\sqrt{b_{1}^{2}+b_{2}^{2}}}$

To obtain the most precise estimates of heading and elevation, use needs to be made of the very precise carrier phase data. The inclusion of the carrier phase data into the model accounts for the presence of the unknown integer ambiguity vector $a$ in (1).

\subsection{Integer least squares}

To solve for the unknown parameter vectors $a$ and $b$, we apply the estimation principle of least-squares (LS) to model (1). This gives the minimization problem

$$
\min _{a, b}\|y-A a-B b\|_{Q_{y y}}^{2}, \quad a \in \mathbb{Z}^{n}, b \in \mathbb{R}^{p}
$$

where $\|\cdot\|_{Q_{y y}}^{2}=(\cdot)^{\mathrm{T}} Q_{y y}^{-1}(\cdot)$. This minimization problem, first introduced in (Teunissen 1993), is called a (mixed) integer least-squares (ILS) problem.

Before presenting the solution to (3), we first consider two simpler, but related, solutions, namely the case that $a$ is known and the case that $a$ is completely unknown. If we assume $a$ known, then the problem to be solved becomes $\min _{b \in \mathbb{R}^{p}}\|y-A a-B b\|_{Q_{y y}}^{2}$, which has the LS-solution

$\hat{b}(a)=\left(B^{\mathrm{T}} Q_{y y}^{-1} B\right)^{-1} B^{\mathrm{T}} Q_{y y}^{-1}(y-A a)$ 
The notation $\hat{b}(a)$ is used to show the dependence on $a$ and to emphasize that we consider $\hat{b}(\cdot)$ to be a function.

Now we consider the case that $a$ is completely unknown. Then the problem to be solved becomes $\min _{a \in \mathbb{R}^{n}, b \in \mathbb{R}^{p}} \| y-$ $A a-B b \|_{Q_{y y}}^{2}$, the solution of which follows from solving the normal equations

$$
\left[\begin{array}{ll}
A^{\mathrm{T}} Q_{y y}^{-1} A & A^{\mathrm{T}} Q_{y y}^{-1} B \\
B^{\mathrm{T}} Q_{y y}^{-1} A & B^{\mathrm{T}} Q_{y y}^{-1} B
\end{array}\right]\left[\begin{array}{l}
\hat{a} \\
\hat{b}
\end{array}\right]=\left[\begin{array}{l}
A^{\mathrm{T}} Q_{y y}^{-1} y \\
B^{\mathrm{T}} Q_{y y}^{-1} y
\end{array}\right]
$$

This solution is given as

$\hat{a}=\left(\bar{A}^{\mathrm{T}} Q_{y y}^{-1} \bar{A}\right)^{-1} \bar{A}^{\mathrm{T}} Q_{y y}^{-1} y$

$\hat{b}=\left(B^{\mathrm{T}} Q_{y y}^{-1} B\right)^{-1} B^{\mathrm{T}} Q_{y y}^{-1}(y-A \hat{a})$

where $\bar{A}=P_{B}^{\perp} A, P_{B}^{\perp}=I_{m}-P_{B}$, and $P_{B}=B\left(B^{\mathrm{T}} Q_{y y}^{-1} B\right)^{-1}$ $B^{\mathrm{T}} Q_{y y}^{-1}$. With respect to the metric $Q_{y y}$, matrix $P_{B}$ is the projector that projects orthogonally onto the range space of $B$. The solution (6) is referred to as the float solution of the GNSS model (1).

The baseline solutions, $\hat{b}(a)$ of (4) and $\hat{b}$ of (6), and their variance matrices, are related as

$$
\begin{aligned}
\hat{b}(a) & =\hat{b}-Q_{\hat{b} \hat{a}} Q_{\hat{a} \hat{a}}^{-1}(\hat{a}-a) \\
Q_{\hat{b}(a) \hat{b}(a)} & =Q_{\hat{b} \hat{b}}-Q_{\hat{b} \hat{a}} Q_{\hat{a} \hat{a}}^{-1} Q_{\hat{a} \hat{b}}
\end{aligned}
$$

Note, assuming $a$ known, that $\hat{b}(a)$ is more precise than $\hat{b}$, i.e. $Q_{\hat{b}(a) \hat{b}(a)} \leq Q_{\hat{b} \hat{b}}$. In case of real time kinematic (RTK) GNSS, this difference is very significant, since $Q_{\hat{b}(a) \hat{b}(a)}$ is then driven by the very precise carrier phase data, while $Q_{\hat{b} \hat{b}}$ is dominated by the relatively poor precision of the code data. In addition, note that $\hat{b}=\hat{b}(\hat{a})$.

In our case, cf. (3), the ambiguity vector $a$ is neither known nor completely unknown. The unknown vector $a$ is namely known to be integer $a \in \mathbb{Z}^{n}$. To find the corresponding LS-solution, we first introduce a useful decomposition of the objective function with the help of (4) and (6). According to Teunissen (1993), we can use $\hat{a}$ and $\hat{b}(a)$ to orthogonally decompose the objective function of (3) as

$$
\begin{aligned}
\|y-A a-B b\|_{Q_{y y}}^{2}= & \|\hat{e}\|_{Q_{y y}}^{2}+\|\hat{a}-a\|_{Q_{\hat{a} \hat{a}}}^{2} \\
& +\|\hat{b}(a)-b\|_{Q_{\hat{b}(a) \hat{b}(a)}^{2}}^{2}
\end{aligned}
$$

where $\hat{e}=y-A \hat{a}-B \hat{b}$ is the float LS residual vector, and $Q_{\hat{a} \hat{a}}=\left(\bar{A}^{\mathrm{T}} Q_{y y}^{-1} \bar{A}\right)^{-1}$ and $Q_{\hat{b}(a) \hat{b}(a)}=\left(B^{\mathrm{T}} Q_{y y}^{-1} B\right)^{-1}$ are the variance matrices of $\hat{a}$ and $\hat{b}(a)$, respectively. From the orthogonal decomposition (8), it is clear that the third term on the right side can be made zero for any $a \in \mathbb{Z}^{n}$; simply choose $b$ equal to $\hat{b}(a)$. The solution to the ILS minimization problem (3) follows therefore as

$\check{a}=\arg \min _{a \in \mathbb{Z}^{n}}\|\hat{a}-a\|_{Q_{\hat{a} \hat{a}}}^{2}$ and $\check{b}=\hat{b}(\check{a})$

These (mixed) ILS-solutions are referred to as the fixed solutions of the GNSS model (1).
The computation of $\check{a}$ involves a search for the integer vector that is closest to $\hat{a}$ in the metric of $Q_{\hat{a} \hat{a}}$. This search is trivial when matrix $Q_{\hat{a} \hat{a}}$ is diagonal, in which case the solution is found from a componentwise integer rounding of the float solution $\hat{a}$. In the case of GNSS, however, matrix $Q_{\hat{a} \hat{a}}$ is nondiagonal and the search becomes nontrivial. The integer minimizer $\breve{a}$ can then be computed efficiently with the LAMBDA method (Teunissen 1993, 1995).

\subsection{Model strength}

Once the ILS solution $\check{a}$ has been computed, the baseline solution $\check{b}$ follows from substituting $\check{a}$ for $a$ in (4). To take full advantage of the very high precision of the carrier phase data, the uncertainty in $\check{a}$ needs to be as small as possible. Only then will the precision of the fixed solution $\breve{b}$ be comparable to the high precision of $\hat{b}(a)$. The uncertainty in $\check{a}$ is negligibly small if the probability of correct integer ambiguity estimation, the so-called ambiguity success rate, is sufficiently close to 1 . The success rate is determined by the strength of the underlying GNSS model; the stronger the model, the higher the success rate. Clearly, the strength of the GNSS model improves when the number of tracked satellites gets larger, when the measurement precision improves, when the number of measurement epochs increases, or when the number of used frequencies gets larger.

It is well-known that dual-frequency, short-baseline RTK ambiguity resolution is possible. It is therefore also possible to obtain precise GNSS RTK Compass readings, when two or more frequencies are observed. For these cases, one can therefore rely on the standard LAMBDA method for successful ambiguity resolution. However, successful ambiguity resolution is generally not possible if only single-frequency data is observed. This is shown in Table 1. This table shows typical values of single-epoch, single-frequency, short-baseline, ILS success rates for different measurement precisions and different number of tracked satellites. It shows that single-frequency, single-epoch ambiguity resolution is not possible when using the standard GNSS model (1), unless the number of tracked satellites is high and the code precision is very good. Hence, a further strengthening of the

\begin{tabular}{|c|c|c|c|}
\hline \multirow{2}{*}{$\begin{array}{l}\sigma_{\phi}(\mathrm{mm}) \\
\sigma_{p}(\mathrm{~cm})\end{array}$} & \multicolumn{3}{|l|}{3} \\
\hline & 30 & 15 & 5 \\
\hline$N=5$ & 3.3 & 19.1 & 86.7 \\
\hline$N=6$ & 24.8 & 66.7 & 96.9 \\
\hline$N=7$ & 50.2 & 79.7 & 99.5 \\
\hline$N=8$ & 86.2 & 94.5 & 99.9 \\
\hline
\end{tabular}
GNSS model is needed if one wants to achieve successful,

Table 1 Single-frequency, single-epoch, short-baseline, ILS success rates $(\%)$ for different measurement precision (undifferenced $\sigma_{\phi}$ and $\left.\sigma_{p}\right)$ and different number of tracked satellites $(N)$ 
single-frequency, epoch-by-epoch ambiguity resolution. A practical way of strengthening the GNSS model in the case of attitude determination is to make use of the known baseline length. In the following section, it will be shown how this information can be integrated rigorously into the ambiguity objective function.

\section{The GNSS compass model}

\subsection{Constrained integer least squares}

If we may assume that the two GNSS antennas are firmly attached to the rigid body of the moving platform, the constant length of the baseline vector may be determined a priori. This allows one to strengthen the GNSS model (1) by including the additional constraint $\|b\|=l$, with $l$ the given baseline length (to denote the unweighted norm, we use instead of $\|\cdot\|_{I}$, the simpler notation $\left.\|\cdot\|\right)$. The use of the baseline length information is of course not new, but our model formulation and method of solution are new. In the literature, the baseline information is usually used to check whether or not the candidate baselines satisfy the given baseline length. It is therefore not included as a rigorous constraint in the estimation principle (see e.g. Park et al. 1996; Hayward et al.; DeLorenzo et al. 2004; Monikes et al. 2005; Wang et al. 2009a).

If we want to include the a priori given baseline information as a constraint, we need to extend the standard GNSS model (1) to

$$
\begin{aligned}
& \mathrm{E}(y)=A a+B b,\|b\|=l, a \in \mathbb{Z}^{n}, b \in \mathbb{R}^{p}, \\
& \mathrm{D}(y)=Q_{y y}
\end{aligned}
$$

This nonlinearly constrained model will be referred to as the GNSS Compass model. It is a linear(ized) GNSS model with a nonlinear constraint on the baseline vector.

Again we apply the LS estimation principle. This time, however, we need to take care of two types of constraints: the integer constraints on the ambiguities, $a \in \mathbb{Z}^{n}$, and the length constraint on the baseline vector, $\|b\|=l$. The latter constraint implies that the baseline vector is constrained to lie on a sphere with radius $l$. We denote this sphere as $\mathbb{S}_{l}=\left\{b \in \mathbb{R}^{p} \mid \quad\|b\|=l\right\}$. With the baseline length constraint included, the LS minimization problem becomes

$$
\min _{a \in \mathbb{Z}^{n}, b \in \mathbb{S}_{l}}\|y-A a-B b\|_{Q_{y y}}^{2}
$$

This LS-problem is coined a quadratically constrained (mixed) integer least-squares (QC-ILS) problem (Park and Teunissen 2003).

To determine the solution of (11), we again make use of the orthogonal decomposition (8). The minimization problem (11) can then be formulated as

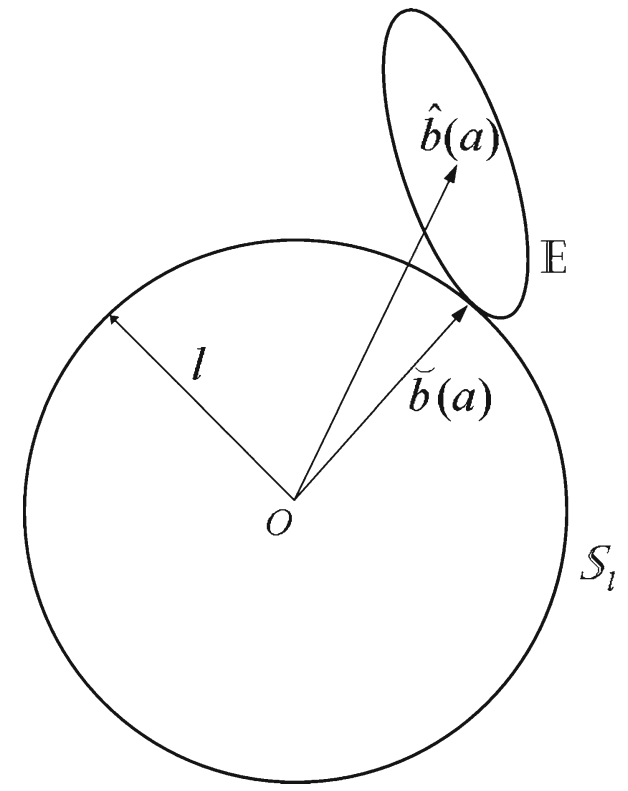

Fig. 1 The geometry of minimizing $\|\hat{b}(a)-b\|_{Q_{\hat{b}(a) \hat{b}(a)}^{2}}^{2}$ for $b \in \mathbb{S}_{l}$

$$
\begin{aligned}
& \min _{a \in \mathbb{Z}^{n}, b \in \mathbb{S}_{l}}\|y-A a-B b\|_{Q_{y y}}^{2}=\|\hat{e}\|_{Q_{y y}}^{2} \\
& +\min _{a \in \mathbb{Z}^{n}}\left(\|\hat{a}-a\|_{Q_{\hat{a} \hat{a}}}^{2}+\min _{b \in \mathbb{S}_{l}}\|\hat{b}(a)-b\|_{Q_{\hat{b}(a) \hat{b}(a)}^{2}}\right)
\end{aligned}
$$

Note that now the third term on the right side does not vanish anymore. This is due to the presence of the baseline length constraint $b \in \mathbb{S}_{l}$. We denote the minimizer of the third term as

$\check{b}(a)=\arg \min _{b \in \mathbb{S}_{l}}\|\hat{b}(a)-b\|_{Q_{\hat{b}(a) \hat{b}(a)}^{2}}$

It is the vector on the sphere of radius $l$ that has smallest distance to $\hat{b}(a)$, where distance is measured with respect to the metric as defined by the variance matrix $Q_{\hat{b}(a) \hat{b}(a)}$. Recall that $\hat{b}(a)$ (c.f. 4 ) is the conditional baseline solution (conditioned on assuming $a$ known). The solution $\breve{b}(a)$, having length $\|\breve{b}(a)\|=l$, is therefore the baseline-length constrained, conditional baseline solution. Geometrically, it can be depicted as the point where the ellipsoid $\mathbb{E}=\{b \in$ $\mathbb{R}^{p} \mid\|\hat{b}(a)-b\|_{Q_{\hat{b}(a) \hat{b}(a)}^{2}}^{2}=$ constant $\}$ just touches the sphere $\mathbb{S}_{l}$ (see Fig. 1).

If we substitute $\breve{b}(a)$ for $b$ in the objective function of (13), it follows that the minimizers $\breve{a}$ and $\breve{b}$ of the QC-ILS problem (13) are given as

$\check{a}=\arg \min _{a \in \mathbb{Z}^{n}}\left(\|\hat{a}-a\|_{Q_{\hat{a} \hat{a}}}^{2}+\|\hat{b}(a)-\check{b}(a)\|_{Q_{\hat{b}(a) \hat{b}(a)}}^{2}\right)$ and

$\check{b}=\check{b}(\check{a})$

These (mixed) QC-ILS solutions are referred to as the fixed solutions of the GNSS Compass model (10) (the fact that we use the same notation as used for the fixed solutions of the 
standard GNSS model (1) should not be a cause of confusion).

To get a first appreciation of the additional complexity that the IAR of the GNSS compass model brings, we compare the QC-ILS ambiguity objective function,

$$
F(a)=\|\hat{a}-a\|_{Q_{\hat{a} \hat{a}}}^{2}+\|\hat{b}(a)-\check{b}(a)\|_{Q_{\hat{b}(a) \hat{b}(a)}^{2}}
$$

(cf. 15), with that of our earlier ILS ambiguity objective function, $E(a)=\|\hat{a}-a\|_{Q_{\hat{a} \hat{a}}}^{2}$ (cf. 9). First note that $F(a)$, in contrast to $E(a)$, not only depends on the ambiguity solution $\hat{a}$, but also on the baseline solution $\hat{b}$. Thus both $\hat{a}$ and $\hat{b}$ are needed as input for the integer minimizer of $F(a)$. Also note that $F(a)$, in contrast to $E(a)$, is nonquadratic in $a$. Hence, its contour surfaces will not be ellipsoidal, thus complicating the integer search for $\check{a}$.

$F(a)$ differs from $E(a)$ by a term that measures the distance, in the metric of $Q_{\hat{b}(a) \hat{b}(a)}$, between $\hat{b}(a)$ and the sphere $\mathbb{S}_{l}$. Hence, potential integer candidates $a \in \mathbb{Z}^{n}$ are now not only downweighted if they are further away from the float solution $\hat{a}$, as is the case with $E(a)$, but also if their corresponding conditional baseline $\hat{b}(a)$ is further apart from the sphere $\mathbb{S}_{l}$. It is this additional penalty in the objective function that allows for higher success rates.

Finally, note that the presence of the second term makes the evaluation of $F(a)$ more time consuming than that of $E(a)$. Its presence implies that $\breve{b}(a)$ of (14) needs to be computed every time $F(a)$ is evaluated. Since the computation of the minimizer $\breve{b}(a)$ is already nontrivial by itself, the presence of this second term is a potential threat for the computational efficiency of the integer search. In Sect. 5, we come back to this issue and we show how the evaluation of the ambiguity objective function can be handled efficiently.

\subsection{Other ambiguity objective functions}

Although our integer ambiguity objective function $F(a)$ is new, other least-squares type ambiguity objective functions have been used in the literature for the GNSS compass problem. It is, therefore, important to understand in what way they differ from our ambiguity objective function.

As mentioned earlier, the use of the baseline length constraint $\|b\|=l$ is not new. All of the existing methods also try, in one way or another, to make use of this constraint, so as to aid the IAR process. However, none of these methods have rigorously incorporated this constraint into the constrained ILS principle. In Han and Rizos (1999), the unconstrained ILS principle is applied with the use of an improved float solution. They first use the baseline length constraint to obtain an improved float solution and then use the standard LAMBDA method to solve for the integer ambiguities as in (9). Also in Dai et al. (2004), Kuylen et al. (2005), Hauschild and Montenbruck (2007), and Hauschild et al. (2008), the standard LAMBDA method is used. Although good results are reported, the baseline constraints are still not used to their fullest. They are used to improve the float solution and/or to check the validity of the integer solution.

Proposals that explicitly incorporate the baseline constraint into the ambiguity search, can be found in Park and Kim (1998), Monikes et al. (2005), Povalyaev et al. (2006) and Wang et al. (2009b). As a result a quadratic inequality constraint is found for the ambiguities. In Park and Kim (1998), Monikes et al. (2005) and Wang et al. (2009b), the quadratic inequality is obtained after the ambiguities have been divided into a primary and secondary set. Hence, as correctly pointed out by Povalyaev et al. (2006), not all ambiguities are incorporated into the inequality constraint and not all information is used in the formation of the inequality constraint. As shown below, this ad hoc division into so-called primary and secondary ambiguity sets is also not needed to construct the quadratic inequality.

Although these latter contributions do use the baseline constraint to explicitly constrain the ambiguity search space, they still do not result in rigorously solving the properly constrained ILS problem. In fact, as we will now show, they still aim at integer minimizing the standard ambiguity objective function, but now in a reduced search space. The idea of these publications is as follows. Since $\|b\|=l$ and $\hat{b}(a)$ is a very precise estimator, one can expect the length of $\hat{b}(a)$ to be very close to $l$, provided $a$ is the correct integer ambiguity vector. Thus one would expect that the correct integer ambiguity vector lies within a set as

$$
\mathbb{C}=\left\{a \in \mathbb{Z}^{n} \mid(l-\epsilon)^{2} \leq\|\hat{b}(a)\|^{2} \leq(l+\epsilon)^{2}\right\}
$$

for some user-defined positive tolerance value $\epsilon$.

The set (17) can be described geometrically as a cylindrical tube. To see this, we first show how $\|\hat{b}(a)\|^{2}$ can be written as a quadratic form in the ambiguity vector $a \in \mathbb{Z}^{n}$. If we write (4) as $\hat{b}(a)=M\left(a_{0}-a\right)$, with the $p \times n$ matrix $M=\left(B^{\mathrm{T}} Q_{y y}^{-1} B\right)^{-1} B^{\mathrm{T}} Q_{y y}^{-1} A$, the $p$-vector $m=\left(B^{\mathrm{T}} Q_{y y}^{-1} B\right)^{-1} B^{\mathrm{T}} Q_{y y}^{-1} y$ and the $n$-vector $a_{0}=M^{\mathrm{T}}\left(M M^{\mathrm{T}}\right)^{-1} m$, we obtain

$\|\hat{b}(a)\|^{2}=\left(a_{0}-a\right)^{\mathrm{T}} M^{\mathrm{T}} M\left(a_{0}-a\right)$

Since matrix $M$ is of order $p \times n$ and rank $p \leq n$, the $n \times n$ matrix $M^{\mathrm{T}} M$ is also of rank $p \leq n$. Thus $M^{\mathrm{T}} M$ has $p$ positive eigenvalues and $n-p$ zero eigenvalues. This implies that the set $\mathbb{C}$, for $\epsilon=0$, is a degenerate ellipsoidal surface, having $p$ axes of finite length and $n-p$ axes of infinite length. It is therefore a cylindrical surface in $\mathbb{R}^{n}$ having a $p$-dimensional ellipsoidal base. For $\epsilon \neq 0$, one obtains two such cylindrical surfaces and the set $\mathbb{C}$ becomes then the region in between these two surfaces, i.e. a cylindrical tube.

In Monikes et al. (2005) and Povalyaev et al. (2006), the standard (unconstrained) ambiguity objective function 
is integer minimized over the restricted set $\mathbb{C}$. Their minimization problem can therefore be formulated as

$\tilde{a}=\arg \min _{a \in \mathbb{C} \cap \mathbb{Z}^{n}}\|\hat{a}-a\|_{Q_{\hat{a} \hat{a}}}^{2}$

Hence, they still integer minimize the quadratic form $\| \hat{a}-$ $a \|_{Q_{\hat{a} \hat{a}}}^{2}$, but now over the region of the cylindrical tube $\mathbb{C} \subset$ $\mathbb{Z}^{n}$, instead over the complete integer space $\mathbb{Z}^{n}$, as is done in the unconstrained ILS case (9). In Park and Kim (1998) and Wang et al. (2009b), a similar problem is solved, but then only based on the so-called primary set of ambiguities. To speed up the computation of (19), Monikes et al. (2005) and Wang et al. (2009b) also use the decorrelation property of the LAMBDA method.

The ambiguity objective function of (19), although of the ILS type, is clearly different from both that of the unconstrained ILS problem, (9), and the constrained ILS problem, (15). Hence, in general, their solutions will also differ. Although the solution of (19) is often an improvement over earlier solutions given in the literature, the underlying principle still lacks rigor. There is no rigorous derivation from first principles that produces (19). Although the step from $\|b\|=l$ to the boundedness of $\|\hat{b}(a)\|$ (cf. 17) may make sense, it is not a derivation. In fact, contrary to (15), the solution produced by (19) does not even use the fact that $\|b\|=l$. Furthermore, with (19), the integer ambiguity solution is required to lie in $\mathbb{C}$, but the size of it, as determined by $\epsilon$, is usually chosen in an ad hoc manner.

\section{Global and local ellipsoidal search}

Although the ambiguity objective function $F(a)$ (cf. 16) is nonquadratic, it would be helpful if its integer minimizer could still be obtained by means of standard ambiguity resolution methods. Since the standard methods have been devised for quadratic objective functions, we now discuss the potential of using an ellipsoidal search for integer minimizing $F(a)$. Two such ellipsoidal search strategies will be presented, a global one and a local one.

\subsection{Weighted constrained integer least squares}

To make our results of the present and following sections valid for both the unconstrained and constrained GNSS models, (1) and (10), we assume from now on that the baseline length constraint is weighted. One may even view this as a more realistic approach. After all, the given baseline length $l$ will usually be the result of an a priori measurement. If we consider the baseline length as an observable, the 'baseline length constraint' takes the form of a nonlinear observation equation $\mathrm{E}(l)=\|b\|$, with variance $\mathrm{D}(l)=\sigma_{l}^{2}$. The integer least-squares solution of this extended model is given by the following Theorem.

Theorem 1 Let $\check{a} \in \mathbb{Z}^{n}$ and $\check{b} \in \mathbb{R}^{p}$ denote the LS parameter solution of the model

$$
\begin{aligned}
& \mathrm{E}(y)=A a+B b, \quad \mathrm{D}(y)=Q_{y y}, a \in \mathbb{Z}^{n} \\
& \mathrm{E}(l)=\|b\|, \quad \mathrm{D}(l)=\sigma_{l}^{2}, b \in \mathbb{R}^{p}
\end{aligned}
$$

where $y$ and $l$ are assumed uncorrelated. Then

$\check{a}=\arg \min _{a \in \mathbb{Z}^{n}} F(a), \quad \check{b}=\arg \min _{b \in \mathbb{R}^{p}} H(\check{a}, b)$

with the ambiguity objective function $F(a)$ and the conditional baseline objective function $H(a, b)$ given as

$$
\begin{aligned}
F(a) & =\|\hat{a}-a\|_{Q_{\hat{a} \hat{a}}}^{2}+\min _{b \in \mathbb{R}^{p}} H(a, b) \\
H(a, b) & =\|\hat{b}(a)-b\|_{Q_{\hat{b}(a) \hat{b}(a)}}^{2}+\sigma_{l}^{-2}(l-\|b\|)^{2}
\end{aligned}
$$

Proof Application of the LS principle to (20) gives the minimization problem

$$
\min _{a \in \mathbb{Z}^{n}, b \in \mathbb{R}^{p}}\left\{\|y-A a-B b\|_{Q_{y y}}^{2}+\sigma_{l}^{-2}(l-\|b\|)^{2}\right\}
$$

By making use of the orthogonal decomposition (8), this minimization problem can be formulated as

$$
\begin{aligned}
& \min _{a \in \mathbb{Z}^{n}, b \in \mathbb{R}^{p}}\|y-A a-B b\|_{Q_{y y}}^{2} \\
& =\min _{a \in \mathbb{Z}^{n}, b \in \mathbb{R}^{p}}\left(\|\hat{e}\|_{Q_{y y}}^{2}+\|\hat{a}-a\|_{Q_{\hat{a} \hat{a}}}^{2}+H(a, b)\right) \\
& =\|\hat{e}\|_{Q_{y y}}^{2}+\min _{a \in \mathbb{Z}^{n}, b \in \mathbb{R}^{p}}\left(\|\hat{a}-a\|_{Q_{\hat{a} \hat{a}}}^{2}+H(a, b)\right) \\
& =\|\hat{e}\|_{Q_{y y}}^{2}+\min _{a \in \mathbb{Z}^{n}}\left(\|\hat{a}-a\|_{Q_{\hat{a} \hat{a}}}^{2}+\min _{b \in \mathbb{R}^{p}} H(a, b)\right)
\end{aligned}
$$

This shows indeed that $\breve{a}$ is given as the integer minimizer of $F(a)$ and that $\breve{b}$ is given as the minimizer of $H(\check{a}, b)$.

Compare (23) with the ILS solution (9) and with the QCILS solution (15). Although we used the same notation $\check{a}, \breve{b}$ and $\breve{b}(a)$ as before, this should not be a cause for confusion. For $\sigma_{l}^{2} \rightarrow \infty$, the weight of the baseline length constraint reduces to zero and the solution of (23) reduces to that of (9). In case of the other extreme, $\sigma_{l}^{2} \rightarrow 0$, the weight of the baseline length enforces a hard constraint and the solution of (23) reduces to that of (15).

The baseline solution from which the final compassinformation is derived is $\breve{b}$ (cf. 21). Similar baseline computations, namely

$\check{b}(a)=\arg \min _{b \in \mathbb{R}^{p}} H(a, b)$

are needed when evaluating the ambiguity objective function $F(a)$. Since $H(a, b)$ (cf. 22$)$ is the least-squares objective function of the nonlinear model $\mathrm{E}(\hat{b}(a))=b, \mathrm{E}(l)=\|b\|$, 
with $y$ and $l$ uncorrelated and $\mathrm{D}(\hat{b}(a))=Q_{\hat{b}(a) \hat{b}(a)}, \mathrm{D}(l)=$ $\sigma_{l}^{2}$, methods for solving nonlinear least-squares problems can be used for computing $\breve{b}(a)$ and $\breve{b}=\breve{b}(\breve{a})$. These methods are, for instance, the iterative descent methods (e.g. the steepest descent method, the Gauss-Newton method, or the Newton method) or globally convergent methods based on the singular value decomposition (see e.g. Teunissen 1990; Golub and Van Loan 1989).

\subsection{A global ellipsoidal search}

The integer minimizer of $F(a)$ can be computed by means of an integer search in the search space

$\Omega_{F}\left(\chi^{2}\right)=\left\{a \in \mathbb{Z}^{n} \mid F(a) \leq \chi^{2}\right\}$

First one sets the size of the search space by choosing a value for $\chi^{2}$. This value should be such that the search space is nonempty. In order to guarantee that $\Omega_{F}\left(\chi^{2}\right)$ is nonempty, one can choose $\chi^{2}=F(z)$ for some $z \in \mathbb{Z}^{n}$. A preferable choice of $z$ is one that returns a small enough value for $\chi^{2}$.

After the size of the search space is set, one collects all integer vectors that lie inside $\Omega_{F}\left(\chi^{2}\right)$ and from this set one then selects the integer vector that returns the smallest value for $F(a)$. This integer vector will be the solution sought, i.e. the integer minimizer of $F(a)$. Thus the global search for the integer minimizer $\breve{a}$ has the following components:

1. Set size: choose small $\chi^{2}$ such that $\Omega_{F}\left(\chi^{2}\right)$ nonempty.

2. Enumerate: find all integer vectors inside $\Omega_{F}\left(\chi^{2}\right)$.

3. Minimize: select $\check{a}$ such that $F(\check{a}) \leq F(a)$ for all $a \in$ $\Omega_{F}\left(\chi^{2}\right)$.

Since the search space (26) is nonellipsoidal, one cannot apply the standard search strategies directly. This complication can be circumvented by working with an ellipsoidal search space instead. But in order to ensure that the global minimizer is included in the enumeration, the ellipsoidal search space needs to encompass (26). Since $F(a) \geq E(a)=$ $\|\hat{a}-\check{a}\|_{Q_{\hat{a} \hat{a}}}^{2}$ for all $a$, this is true for the ellipsoidal set (see Fig. 2),

$\Omega_{E}\left(\chi^{2}\right)=\left\{a \in \mathbb{Z}^{n} \mid E(a) \leq \chi^{2}\right\} \supset \Omega_{F}\left(\chi^{2}\right)$

Hence, the enumeration now consists of finding the integer candidates in the ellipsoidal set $\Omega_{E}\left(\chi^{2}\right)$ that also lie in $\Omega_{F}\left(\chi^{2}\right)$. Finding the integer candidates in $\Omega_{E}\left(\chi^{2}\right)$ can be done efficiently with the LAMBDA method. Checking whether such $z \in \Omega_{E}\left(\chi^{2}\right)$ also lies in $\Omega_{F}\left(\chi^{2}\right)$, amounts then to checking the inequality

$G(z)=\min _{b \in \mathbb{R}^{p}} H(z, b) \leq \chi^{2}-\|\hat{a}-z\|_{Q_{\hat{a} \hat{a}}}^{2}$

If this inequality is satisfied, then $F(z) \leq \chi^{2}$ and thus $z \in$ $\Omega_{F}\left(\chi^{2}\right)$.

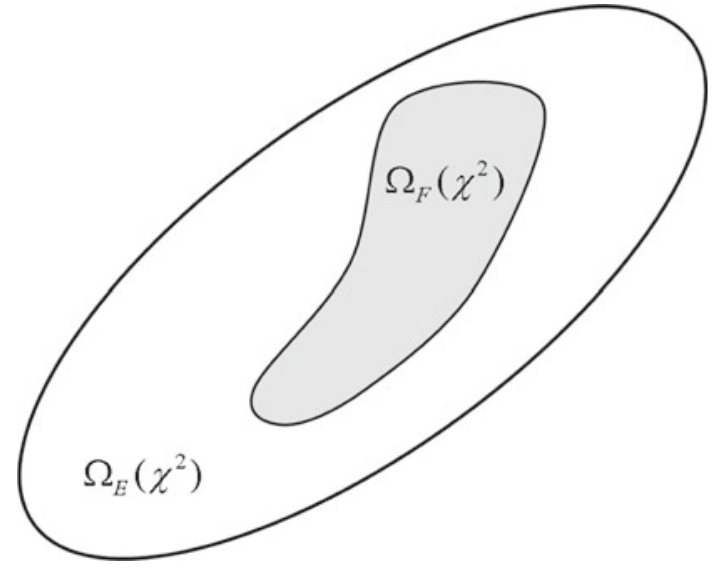

Fig. 2 Two-dimensional example of $\Omega_{F}\left(\chi^{2}\right) \subset \Omega_{E}\left(\chi^{2}\right)$

Clearly this ellipsoidal-based search is rigorous, simple and rather straightforward to apply. This search is, therefore, an attractive method for finding $\breve{a}$, provided it can be performed in a timely manner. The search becomes inefficient though if the ellipsoidal search space contains too many integer vectors. Both the enumeration and minimization will then contribute to a slow down of the computational process. The larger the search space, the more integer vectors need to be enumerated and the more often the function $F(a)$ needs to be evaluated.

A too large search space $\Omega_{E}\left(\chi^{2}\right)$ can be avoided if one is able to compute a small enough value for $\chi^{2}$. This is possible if the underlying unconstrained GNSS model has sufficient strength. This is the case, for example, with short-baseline, multi-frequency models. For such models, bootstrapping (or rounding) the float solution $\hat{a}$ usually already gives an integer close to the sought for integer solution (Teunissen 1998). Such a bootstrapped (or rounded) integer vector can then be used to compute $\chi^{2}$. Thus for GNSS models that have sufficient strength, the above LAMBDA-based search is attractive.

This is generally not the case however, for models that are based on single-frequency, single-epoch data. These models are generally too weak to permit such a straightforward approach as described above. To understand this, we first show that for such models, the second term, $G(a)=$ $\min _{b} H(a, b)$, in $F(a)=E(a)+G(a)$, is usually some orders of magnitude larger than the first term, $E(a)=$ $\|\hat{a}-a\|_{Q_{\hat{a} \hat{a}}}^{2}$. This is due to the different weighting in the terms of $F(a)$. The ambiguity residual in $E(a)$ is weighted by the inverse of the ambiguity variance matrix, while the residuals of $G(a)$ are weighted by the inverse conditional baseline variance matrix and the inverse of $\sigma_{l}^{2}$, respectively. As the following example shows, these weights differ by some orders of magnitude.

In the single epoch, single frequency case, the design matrices $A$ and $B$ of the model (10) are structured as 
$A=\left[\lambda I_{n}, 0\right]^{\mathrm{T}}$ and $B=\left[G^{\mathrm{T}}, G^{\mathrm{T}}\right]^{\mathrm{T}}$, with $\lambda$ the wavelength and $G$ the geometry matrix, that contains the unit direction vectors to the satellites. With $\sigma_{\phi}^{2} Q$ and $\sigma_{p}^{2} Q$ being the variance matrices of the single-frequency DD phase and code data, respectively, the ambiguity variance matrix $Q_{\hat{a} \hat{a}}=\left(\bar{A}^{\mathrm{T}} Q_{y y}^{-1} \bar{A}\right)^{-1}$ follows as

$$
\begin{aligned}
Q_{\hat{a} \hat{a}} & =\frac{\sigma_{p}^{2}}{\lambda^{2}}\left(\frac{\sigma_{\phi}^{2}}{\sigma_{p}^{2}} Q+G\left(G^{\mathrm{T}} Q^{-1} G\right)^{-1} G^{\mathrm{T}}\right) \\
& \approx \frac{\sigma_{p}^{2}}{\lambda^{2}} G\left(G^{\mathrm{T}} Q^{-1} G\right)^{-1} G^{\mathrm{T}}
\end{aligned}
$$

and the conditional baseline variance matrix $Q_{\hat{b}(a) \hat{b}(a)}=$ $\left(B^{\mathrm{T}} Q_{y y}^{-1} B\right)^{-1}$ follows as

$$
\begin{aligned}
Q_{\hat{b}(a) \hat{b}(a)} & =\frac{\sigma_{\phi}^{2}}{1+\sigma_{\phi}^{2} / \sigma_{p}^{2}}\left(G^{\mathrm{T}} Q^{-1} G\right)^{-1} \\
& \approx \sigma_{\phi}^{2}\left(G^{\mathrm{T}} Q^{-1} G\right)^{-1}
\end{aligned}
$$

This shows, since $\sigma_{\phi}^{2} \ll \sigma_{p}^{2}$ and $\sigma_{l}^{2}$ is usually chosen very small as well, that for most $a$, the first term $E(a)$ of $F(a)$ will be much smaller than its second term $G(a)$. Thus $F(z) \gg$ $E(z)$ for most $z \in \mathbb{Z}^{n}$. As a consequence, the value $\chi^{2}=$ $F(z)$, which is used to set the size of $\Omega_{E}\left(\chi^{2}\right)$, will be large too. This implies that $\Omega_{E}\left(\chi^{2}\right)$ will contain many integer vectors, most of which will be rejected again by the inequality check (28). Thus, in this case many of the collected integer vectors will be computed with no avail (search halting) and, moreover, for the many integer vectors inside $\Omega_{E}\left(\chi^{2}\right)$, one will have to compute $\breve{b}(a)$, which may considerably slow down the estimation process. The conclusion is therefore, that for the single epoch, single frequency case, an alternative approach is needed for selecting $\chi^{2}$. Moreover, it would be helpful, if, in the evaluation of the integer candidates, one can avoid the necessity of having to compute $\|\hat{b}(a)-\breve{b}(a)\|_{Q_{\hat{b}(a)}}^{2}$ too often.

\subsection{A local ellipsoidal search}

Instead of trying to integer minimize $F(a)$ rigorously, one can also try to solve it in an approximate sense, i.e. by minimizing an approximation to the ambiguity objective function. The idea is therefore to make use of a quadratic approximation, such that standard IAR methods can be applied again. For such a quadratic approximation, the first and second order partial derivatives of the ambiguity objective function are needed. They are given by the following Lemma.

Lemma 1 The gradient vector and the Hessian matrix of the ambiguity objective function $F(a)$ (cf. 22) are given as

$$
\begin{gathered}
\partial_{a} F(a)=-2 \bar{A}^{\mathrm{T}} Q_{y y}^{-1} \bar{A}(\hat{a}-a)-2 A^{\mathrm{T}} Q_{y y}^{-1} B(\hat{b}(a)-\check{b}(a)) \\
\partial_{a a}^{2} F(a)=2 A^{\mathrm{T}} Q_{y y}^{-1} A-2 A^{\mathrm{T}} Q_{y y}^{-1} B \\
\times\left[B^{\mathrm{T}} Q_{y y}^{-1} B+\sigma_{l}^{-2} X(\check{b}(a))\right]^{-1} B^{\mathrm{T}} Q_{y y}^{-1} A
\end{gathered}
$$

with $\hat{b}(a)$ given by (7), $\breve{b}(a)$ by (25) and where $X(b)$ is the $p \times p$ matrix function

$X(b)=P_{b}+\left(1-\frac{l}{\|b\|}\right) P_{b}^{\perp}$

with the orthogonal projectors $P_{b}=b\left(b^{\mathrm{T}} b\right)^{-1} b^{\mathrm{T}}$ and $P_{b}^{\perp}=$ $I_{p}-P_{b}$.

Proof Since the proof is rather lengthy, we only give a sketch. First, the first order and second order partial derivatives of $H(a, b)$ (cf. 22) are determined. Then the gradient and Hessian of $G(a)=\arg \min _{b \in \mathbb{R}^{p}} H(a, b)$ are expressed in the partial derivatives of $H(a, b)$. Combining these results and recognizing that $\partial_{b} H(a, \breve{b}(a))=0$ for all $a \in \mathbb{R}^{n}$, gives (31).

With (31), we still have the option of choosing the point of approximation freely. Here we will choose the real-valued minimizer of $F(a)$ as the point of approximation. Since it is the least-squares solution of the GNSS Compass model (10) without the integer constraints $a \in \mathbb{Z}^{n}$, it is in fact the 'best' float solution one can get. This constrained float solution, denoted as $\bar{a}$ and $\bar{b}$, is related to the unconstrained float solution, $\hat{a}$ and $\hat{b}$, as

$\bar{a}=\hat{a}-Q_{\hat{a} \hat{b}} Q_{\hat{b} \hat{b}}^{-1}(\hat{b}-\bar{b})$

$\bar{b}=\arg \min _{b \in \mathbb{R}^{p}}\left(\|\hat{b}-b\|_{Q_{\hat{b} \hat{b}}}^{2}+\sigma_{l}^{-2}(l-\|b\|)^{2}\right)$

Thus, once $\bar{b}$ has been solved as a nonlinear least-squares problem, only a linear correction on $\hat{a}$ is needed to obtain $\bar{a}$.

We are now in the position to show how the standard IAR methods can be used to solve for the approximate integer minimizer of the ambiguity objective function.

Theorem 2 The integer minimizer of the quadratic approximation of $F(a)$ at $\bar{a}$, is given by the ILS solution

$\check{a}^{\prime}=\arg \min _{a \in \mathbb{Z}^{n}}\|\bar{a}-a\|_{Q}^{2}$

where

$$
\begin{aligned}
Q & =\left[\frac{1}{2} \partial_{a a}^{2} F(\bar{a})\right]^{-1}=Q_{\hat{a} \hat{a}}-Q_{\hat{a} \hat{b}}\left[Q_{\hat{b} \hat{b}}+\sigma_{l}^{2} X(\bar{b})^{-1}\right]^{-1} Q_{\hat{b} \hat{a}} \\
& =Q_{\hat{a}(b) \hat{a}(b)}+Q_{\hat{a} \hat{b}} Q_{\hat{b} \hat{b}}^{-1}\left[Q_{\hat{b} \hat{b}}^{-1}+\sigma_{l}^{-2} X(\bar{b})\right]^{-1} Q_{\hat{b} \hat{b}}^{-1} Q_{\hat{b} \hat{a}}
\end{aligned}
$$


Proof With the use of (31), we develop $F(a)$ in a Taylor series at $\bar{a}$. Since $\partial_{a} F(\bar{a})=0$ (recall that $\bar{a}$ is the minimizer of $F(a)$ ), we obtain the quadratic approximation $F(a) \approx$ $F(\bar{a})+\|\bar{a}-a\|_{Q}^{2}$, which proves (34). The two expressions of (35) follow from applying the well-known matrix inversion Lemma $\left(M-N R^{-1} P\right)^{-1}=M^{-1}+M^{-1} N(R-$ $\left.P M^{-1} N\right)^{-1} P M^{-1}$ to the expression of the Hessian in (31) evaluated at $\bar{a}$, thereby recognizing that $\bar{b}=\check{b}(\bar{a})$.

It follows from (35) that

$Q_{\hat{a}(b) \hat{a}(b)} \leq\left[\frac{1}{2} \partial_{a a}^{2} F(a)\right]^{-1} \leq Q_{\hat{a} \hat{a}}$

if $X \geq 0$. These matrix lower and upper bounds are understandable, since $Q_{\hat{a}(b) \hat{a}(b)}$ is the ambiguity variance matrix would $b$ be completely known, while $Q_{\hat{a} \hat{a}}$ is the ambiguity variance matrix for $b$ completely unknown.

The results of the above Theorem can be used in three different ways. First, the ILS solution $\check{a}^{\prime}$ may be used in its own right as a replacement of $\breve{a}$. This is meaningful if one can show that the success rate of $\check{a}^{\prime}$ is already high enough. Second, instead of using $\check{a}^{\prime}$ as a replacement of $\breve{a}$, one may also think of using bootstrapping or rounding on the quadratic approximation. In the case of bootstrapping, one would then integer bootstrap the decorrelated version of $\bar{a}$ using $\left[\frac{1}{2} \partial_{a a}^{2} F(\bar{a})\right]$ as weight matrix (Teunissen 1998). Finally, one may also use $\check{a}^{\prime}$, or its bootstrapped or rounded version, to set the size of the nonellipsoidal search space through $\chi^{2}=$ $F\left(\check{a}^{\prime}\right)$.

Whether the above quadratic approximation can be used as a basis for computing a useful integer solution depends to a large extend on the length of the baseline. The longer the baseline, the better the quadratic approximation. Hence, if the baseline is long enough, one can perform successful, single-frequency, single-epoch ambiguity resolution with the standard LAMBDA method, but now applied to the above quadratic approximation of the GNSS Compass model. Table 2 gives for a typical single-frequency, single-epoch GPS scenario, the ambiguity success rates for different baseline lengths and for different measurement precisions. These

Table 2 Single-frequency, single-epoch, ILS GPS ambiguity success rates $(\%)$ based on a quadratic approximation of $F(a)$ for different measurement precision (undifferenced $\sigma_{\phi}$ and $\sigma_{p}$ ) and for different baseline lengths $(\|b\|)$ using six tracked satellites $\left(\sigma_{l}=0\right)$

\begin{tabular}{llll}
\hline$\sigma_{\phi}(\mathrm{mm})$ & 3 & & \\
\cline { 2 - 4 }$\sigma_{p}(\mathrm{~cm})$ & 30 & 15 & 5 \\
\hline$\|b\|=0.5 \mathrm{~m}$ & 8.2 & 18.2 & 71.8 \\
$\|b\|=1 \mathrm{~m}$ & 8.5 & 23.6 & 87.6 \\
$\|b\|=2 \mathrm{~m}$ & 11.5 & 38.1 & 97.1 \\
$\|b\|=5 \mathrm{~m}$ & 21.4 & 63.7 & 99.8 \\
$\|b\|=20 \mathrm{~m}$ & 53.5 & 94.3 & 99.9 \\
$\|b\|=50 \mathrm{~m}$ & 77.5 & 99.3 & 99.9 \\
\hline
\end{tabular}

results show, for $\sigma_{\phi}=3 \mathrm{~mm}$ and $\sigma_{p}=15 \mathrm{~cm}$, that baseline lengths of $50 \mathrm{~m}$ or longer will give success rates higher than 99\% (for long baselines, the possible lack of platform rigidity may be captured through $\sigma_{l}$ ). Hence, with this approximate method one can not expect instantaneous IAR to be successful for small platforms. For larger platforms however, such as vessels, the method is indeed promising. Moreover, one should keep in mind that as the code precision improves (as is the case with the future GNSSs), the baseline length is allowed to become shorter to achieve the same success rate performance.

\section{A search and shrink strategy using bounding functions}

\subsection{On the geometry of the search space}

We already remarked, that contrary to the contour surfaces of the standard ambiguity objective function, the contour surfaces of $F(a)$ are nonellipsoidal. To get a better insight into this geometry, we consider the contour surfaces of $F(a)$, but now under the simplifying assumption that the conditional baseline variance matrix is diagonal. This allows us to obtain a closed form expression for the second term in $F(a)$, the $b$-minimum of $H(a, b)$ (cf. 22).

Lemma 2 If $Q_{\hat{b}(a) \hat{b}(a)}=\frac{1}{\lambda} I_{p}$ and $\hat{b}(a) \neq 0$, then the $b$-minimizer and the $b$-minimum, respectively, of $H(a, b)=$ $\|\hat{b}(a)-b\|_{Q_{\hat{b}(a) \hat{b}(a)}^{2}}^{2}+\sigma_{l}^{-2}(l-\|b\|)^{2}$, are given as

$\check{b}(a)=\arg \min _{b \in \mathbb{R}^{p}} H(a, b)=\frac{l+\sigma_{l}^{2} \lambda\|\hat{b}(a)\|}{1+\sigma_{l}^{2} \lambda} \frac{\hat{b}(a)}{\|\hat{b}(a)\|}$

and

$\min _{b \in \mathbb{R}^{p}} H(a, b)=\frac{\lambda}{1+\sigma_{l}^{2} \lambda}(l-\|\hat{b}(a)\|)^{2}$

If $\hat{b}(a)=0$, then the minimizer is not unique, but the minimum still is.

Proof Let $\hat{b}(a) \neq 0$. Since the contour surfaces of $\| \hat{b}(a)-$ $b \|\left.\right|_{Q} ^{2}$ are spheres centred at $\hat{b}(a)$ (if $Q=\frac{1}{\lambda}$ ) and the contour surfaces of $\sigma_{l}^{-2}(l-|| b||)^{2}$ are spheres centred at the origin, it follows directly from the geometry of the problem that the $b$-minimizer of $H(a, b)$ must lie in between the origin and $\hat{b}(a)$, on the straight line connecting these two points. Thus $\breve{b}(a)=\mu \hat{b}(a) /\|\hat{b}(a)\|$ for some scalar $\mu$ in the interval $0 \leq \mu \leq\|\hat{b}(a)\|$. Substitution of this expression into $H(a, b)$ reduces the problem to a minimization of a quadratic function in the scalar $\mu$. This $\mu$-minimizer is given as $\hat{\mu}=\frac{l+\sigma_{l}^{2} \lambda\|\hat{b}(a)\|}{1+\sigma_{l}^{2} \lambda}$. Hence, it is the weighted average of $\|\hat{b}(a)\|$ and $l$. This proves (37). The minimum (38) simply follows from substituting the $b$-minimizer into $H(a, b)$. 
If $\hat{b}(a)=0$, then $H(a, b)$ is constant for constant $\|b\|$. Hence, the $b$-minimizer is then not unique, but the minimum still is, $\min _{b \in \mathbb{R}^{p}} H(a, b)=\frac{\lambda}{1+\sigma_{l}^{2} \lambda} l^{2}$.

This Lemma shows that if the conditional baseline variance matrix is a scaled unit matrix, the solution $\breve{b}(a)$ follows from an orthogonal projection of $\hat{b}(a)$ onto the origin centred sphere, with radius equal to the weighted average of $l$ and $\|\hat{b}(a)\|$. For $\sigma_{l}^{2} \rightarrow 0$, the projection is onto the sphere with radius $l$, which corresponds to the hard constraint case. For $\sigma_{l}^{2} \rightarrow \infty$, which corresponds to the unconstrained case, the projection is trivial, since then $\breve{b}(a)=\hat{b}(a)$.

In reality, of course, the actual conditional baseline variance matrix will not be diagonal. Still, for a properly chosen scalar $\lambda$, the above result can be seen as a useful approximation. For instance, the solution (37) may be used to initialize the iterative schemes for solving the nonlinear least-squares problem (25).

A useful choice for $\lambda$ is the average of the eigenvalues of the inverse of $Q_{\hat{b}(a) \hat{b}(a)}$. Thus $\lambda=\frac{1}{p} \operatorname{trace}\left(B^{\mathrm{T}} Q_{y y}^{-1} B\right)$. This choice corresponds to the LS approximation of matrix $B^{\mathrm{T}} Q_{y y}^{-1} B$ by $\lambda I_{p}$ in the Frobenius norm.

Using the closed form expression for the $b$-minimum of $H(a, b)$ in (38), the expression for the ambiguity objective function $F(a)$ simplifies and further insight into the geometry of its search space can be gained.

Theorem 3 If $Q_{\hat{b}(a) \hat{b}(a)}=\frac{1}{\lambda} I_{p}$, then the search space of $F(a)$ simplifies to

$$
\begin{aligned}
& \Omega_{F}\left(\chi^{2}\right)=\left\{a \in \mathbb{Z}^{n} \mid F(a)=E(a)+\min _{b \in \mathbb{R}^{p}} H(a, b) \leq \chi^{2}\right\} \\
& =\left\{a \in \mathbb{Z}^{n} \mid F(a)=E(a)+\mu(l-\|\hat{b}(a)\|)^{2} \leq \chi^{2}\right\} \\
& =\left\{a \in \mathbb{Z}^{n} \mid E(a) \leq \chi^{2} \text { and } L(a)^{2} \leq\left\|a_{0}-a\right\|_{R^{+}}^{2} \leq U(a)^{2}\right\} \\
& \subset \Omega_{E}\left(\chi^{2}\right) \cap \mathbb{C}_{0}\left(\chi^{2}\right)
\end{aligned}
$$

with the subsets

$$
\begin{aligned}
\Omega_{E}\left(\chi^{2}\right) & =\left\{a \in \mathbb{Z}^{n} \mid E(a)=\|\hat{a}-a\|_{Q_{\hat{a} \hat{a}}}^{2} \leq \chi^{2}\right\} \\
\mathbb{C}_{0}\left(\chi^{2}\right) & =\left\{a \in \mathbb{Z}^{n} \mid L_{0}^{2} \leq\left\|a_{0}-a\right\|_{R^{+}}^{2} \leq U_{0}^{2}\right\}
\end{aligned}
$$

and where

$$
\begin{aligned}
& L(a)=2 l-U(a), U(a)=l+\sqrt{\left(\chi^{2}-E(a)\right) / \mu} \\
& R=M^{\mathrm{T}} M, M=\left(B^{\mathrm{T}} Q_{y y}^{-1} B\right)^{-1} B^{\mathrm{T}} Q_{y y}^{-1} A \\
& a_{0}=M^{+} m, m=\left(B^{\mathrm{T}} Q_{y y}^{-1} B\right)^{-1} B^{\mathrm{T}} Q_{y y}^{-1} y \\
& L_{0}=L(\hat{a}), U_{0}=U(\hat{a}), \mu=\lambda /\left(1+\sigma_{l}^{2} \lambda\right)
\end{aligned}
$$

Proof With (38), we have $F(a)=E(a)+\min _{b \in \mathbb{R}^{p}} H(a, b)=$ $E(a)+\mu(l-\|\hat{b}(a)\|)^{2}$. Therefore, $F(a) \leq \chi^{2}$ is equivalent to $(l-\|\hat{b}(a)\|)^{2} \leq\left(\chi^{2}-E(a)\right) / \mu$, or to $L(a)^{2} \leq \| a_{0}-$ $a \|_{R^{+}}^{2} \leq U(a)^{2}$, since $\|\hat{b}(a)\|^{2}=\left(a_{0}-a\right)^{\mathrm{T}} M^{\mathrm{T}} M\left(a_{0}-a\right)$. The last line of (39) follows from observing that $L_{0}^{2} \leq L(a)^{2}$ and $U_{0}^{2} \geq U(a)^{2}$ for all $a$.
To get an appreciation of the search space geometry, consider the one-dimensional case $n=p=1$ first. From (39) follows then that $F(a)$ is formed from two different parabolas, one parabola for the interval $a \leq a_{0}$ and another parabola for the interval $a \geq a_{0}$. This shows, depending on the value of $\chi^{2}$, that the one-dimensional search space $\Omega_{F}\left(\chi^{2}\right)$ may consist of a single interval or of two separate intervals. The latter case may also be viewed as a single interval with a hole in the middle. This type of geometry extends itself to the higher dimensional case too, as can be verified by taking one-dimensional sections of $\Omega_{F}\left(\chi^{2}\right) \subset \mathbb{R}^{n}$. Hence, the search space is not convex and it may even have a hole in it. This hole may be located somewhere in the middle or at the side, in which case the search space becomes somewhat moon-shaped.

Despite the complex geometry of the search space, the last expression of (39) shows that the search space stays confined in the intersection of two well-defined spaces, the intersection of an ellipsoid with a cylindrical tube, namely $\Omega_{E}\left(\chi^{2}\right)$ and $\mathbb{C}_{0}\left(\chi^{2}\right)$, respectively.

\subsection{A nonellipsoidal search and shrink for an approximate integer minimizer}

In Sect. 4.3, we introduced an approximate integer minimizer of $F(a)$ by means of a quadratic approximation. This approach has the advantage that the standard LAMBDA method can be used. To obtain large enough success rates, however, the method requires not too small baselines. In this section, we introduce another approximate integer minimizer, namely the one that integer minimizes $F(a)$ for $Q_{\hat{b}(a) \hat{b}(a)}=\frac{1}{\lambda} I_{p}$. This integer minimizer is thus defined as

$\check{a}^{\prime \prime}=\arg \min _{a \in \mathbb{Z}^{n}}\left(\|\hat{a}-a\|_{Q_{\hat{a} \hat{a}}}^{2}+\frac{\lambda}{1+\sigma_{l}^{2} \lambda}(l-\|\hat{b}(a)\|)^{2}\right)$

This solution will be a good integer approximation of $\check{a}$ (cf. 21), if $Q_{\hat{b}(a) \hat{b}(a)}$ is close to $\frac{1}{\lambda} I_{p}$. Note that the performance of $\check{a}^{\prime \prime}$ is now not dependent on how long the baseline is, as it is the case with the integer approximation $\check{a}^{\prime}$ (cf. 34), but rather on how well the conditional baseline variance matrix can be approximated by a diagonal matrix.

According to Theorem 3, the solution $\breve{a}^{\prime \prime}$ can be obtained by searching for the integer minimzier of $F(a)$ in the intersection of ellipsoid $\Omega_{E}\left(\chi^{2}\right)$ and cylindrical tube $\mathbb{C}_{0}\left(\chi^{2}\right)$. We now describe our search and shrink strategy for computing this approximate integer minimizer. First consider the case $n=p$. The two quadratic forms, $\|\hat{a}-a\|_{Q_{\hat{a} \hat{a}}}^{2}$ and $\left\|a_{0}-a\right\|_{R^{+}}^{2}$, are then both of full rank and can therefore both be written as a sum-of-squares. These sum-ofsquares structures are then used to replace the quadratic form inequalities of (39) by a set of $p$ scalar inequalities, each 
describing a sequential interval (possibly with holes). This is similar to how the sequential search intervals are formulated in the LAMBDA method (Teunissen 1995; de Jonge and Tiberius 1996). The $p$ sequential intervals are denoted as $I\left(a_{1}\right), I\left(a_{2} \mid a_{1}\right), \ldots, I\left(a_{p} \mid a_{1}, \ldots, a_{p-1}\right)$. The interval $I\left(a_{i} \mid a_{1}, \ldots, a_{i-1}\right)$ is an interval for $a_{i}$ and it can be computed once $a_{1}, \ldots, a_{i-1}$ are given. Together, the $p$ scalar intervals describe the same set as the intersection of ellipsoid and cylindrical tube. By working through the intervals sequentially, i.e. starting with $I\left(a_{1}\right)$ and ending with $I\left(a_{p} \mid a_{1}, \ldots, a_{p-1}\right)$, one can collect all integer candidates. To make this search more efficient, one chooses each time that one arrives at the last interval $I\left(a_{p} \mid a_{1}, \ldots, a_{p-1}\right)$, the value of $a_{p}$ such that it minimizes $F(a)$. This allows one to shrink the search space, i.e. compute a smaller value for $\chi^{2}$, and to repeat the search in a smaller search space. This process is repeated until only one integer vector is left, the integer minimizer of $F(a)$.

For $n>p$, one can still use the above procedure, be it that one first needs to find candidate values for the first $n-p$ entries of $a$. They will need to come from the ellipsoid $\Omega_{E}\left(\chi^{2}\right)$, since the cylindrical tube is degenerate in $n-p$ dimensions. Thus $a$ is partitioned as $a=\left(a_{I}^{\mathrm{T}}, a_{I I}^{\mathrm{T}}\right)^{\mathrm{T}}$ and since $\|\hat{a}-a\|_{Q_{\hat{a} \hat{a}}}^{2}=\left\|\hat{a}_{I}-a_{I}\right\|_{Q_{\hat{a}_{I} \hat{a}_{I}}}^{2}+\left\|\hat{a}_{I I}\left(a_{I}\right)-a_{I I}\right\|_{Q_{\hat{a}_{I I \mid I} \hat{a}_{I I \mid I}}^{2} \leq} \leq$ $\chi^{2}$, we first use $\left\|\hat{a}_{I}-a_{I}\right\|_{Q_{\hat{a}_{I} \hat{a}_{I}}}^{2} \leq \chi^{2}$ to find integer candidates $a_{I}$ and then use $\hat{a}_{I I}\left(a_{I}\right)$ to find integer candidates $a_{I I}$ in a similar way as done above for $n=p$. Once a candidate integer vector, say $z=\left(z_{I}^{\mathrm{T}}, z_{I I}^{\mathrm{T}}\right)^{\mathrm{T}}$ is found, the size of the search space is reset, $\chi^{2}=F(z)$, and the process is repeated in the shrunken search space. This process is repeated until only one integer vector is left, the integer minimizer of $F(a)$.

\subsection{Upper/lower bounding of search space}

Both of our approximations of $F(a)$ (cf. Sects. 4.3, 5.2) have the advantage that they avoid the complexity of having to compute $\breve{b}(a)$ (cf. 25) during the search. But they do of course not guarantee a global integer minimizer as result. In order to avoid computing $\breve{b}(a)$ too often and still be able to guarantee that we can find the global minimizer, let us for the moment assume to have functions that are easy to evaluate and that bound $F(a)$ from below and from above:

$$
F_{1}(a) \leq F(a) \leq F_{2}(a)
$$

With such bounding functions correspond the two search spaces

$$
\begin{aligned}
& \Omega_{1}\left(\chi^{2}\right)=\left\{a \in \mathbb{Z}^{n} \mid F_{1}(a) \leq \chi^{2}\right\} \\
& \Omega_{2}\left(\chi^{2}\right)=\left\{a \in \mathbb{Z}^{n} \mid F_{2}(a) \leq \chi^{2}\right\}
\end{aligned}
$$

They bound $\Omega_{F}\left(\chi^{2}\right)$ as

$$
\Omega_{2}\left(\chi^{2}\right) \subset \Omega_{F}\left(\chi^{2}\right) \subset \Omega_{1}\left(\chi^{2}\right)
$$

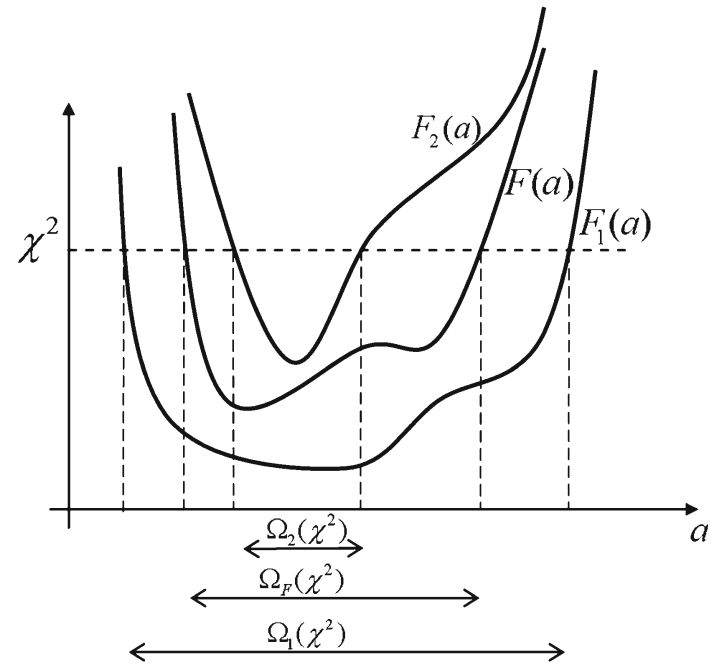

Fig. 3 Lower and upper bounding of objective function $F(a)$ and search space $\Omega_{F}\left(\chi^{2}\right)$

Note that the set ordering is the reverse of the function ordering. Also note that the sizes of the three sets are defined by the same $\chi^{2}$. Thus by varying $\chi^{2}$, all three sets change in size, but the set ordering (45) remains intact (see Fig. 3).

Now recall that the global minimizer $\breve{a}$ is found by evaluating $F(a)$ for all vectors in $\Omega_{F}\left(\chi^{2}\right)$, followed by selecting the vector that returns the smallest function value. Hence, one would like $\Omega_{F}\left(\chi^{2}\right)$ to be small and nonempty. The nonemptiness of $\Omega_{F}\left(\chi^{2}\right)$ guarantees that it contains $\check{a}$, and the smallness helps avoiding a multitude of function evaluations $F(a)$. These two requirements are met if $\Omega_{2}\left(\chi^{2}\right) \subset \Omega_{F}\left(\chi^{2}\right)$ is nonempty and $\Omega_{1}\left(\chi^{2}\right) \supset \Omega_{F}\left(\chi^{2}\right)$ is small.

To obtain a small $\Omega_{1}\left(\chi^{2}\right)$ while ensuring that $\Omega_{2}\left(\chi^{2}\right)$ is nonempty, we compute the smallest $\chi^{2}$ for which $\Omega_{2}\left(\chi^{2}\right)$ is nonempty. Hence, we determine $\chi^{2}$ from the integer minimizer of $F_{2}(a)$. This integer minimizer is determined by means of a search and shrink strategy. Starting with an initial $\chi_{0}^{2}$, we search for an integer vector in the space:

$\Omega_{2}\left(\chi_{0}^{2}\right)=\left\{a \in \mathbb{Z}^{n} \mid F_{2}(a) \leq \chi_{0}^{2}\right\} \subset \Omega_{F}\left(\chi_{0}^{2}\right)$

As soon as such an integer vector is found, say $\tilde{a}$, the space is shrunk to the value $\tilde{\chi}^{2}=F_{2}(\tilde{a})<\chi_{0}^{2}$ and the search continues in this smaller set. In this way the search proceeds rather quickly towards the integer minimizer of $F_{2}(a)$, which we denote as $\check{a}_{2}$. Note that this integer vector is not necessarily the integer minimizer of $F(a)$, but we do know that it lies inside the set

$\Omega_{F}\left(\chi_{1}^{2}\right) \subset \Omega_{1}\left(\chi_{1}^{2}\right)=\left\{a \in \mathbb{Z}^{n} \mid F_{1}(a) \leq \chi_{1}^{2}\right\}$

with $\chi_{1}^{2}=F_{2}\left(\check{a}_{2}\right)$. The sought for minimizer $\check{a}$ is then found as the integer vector of $\Omega_{1}\left(\chi_{1}^{2}\right)$ that returns the smallest value of $F(a)$. 
In principle, different choices for the bounding functions can be made. For instance, it is possible to choose $F_{1}(a)$ and $F_{2}(a)$ as quadratic forms so that $\Omega_{1}\left(\chi^{2}\right)$ and $\Omega_{2}\left(\chi^{2}\right)$ of (45) become ellipsoidal regions. With the search and shrink strategy, this gives the possibility to speed up the global ellipsoidal search strategy of Sect. 4.2.

The goal of working with the bounding sets is that we end up with a set $\Omega_{1}\left(\chi^{2}\right) \supset \Omega_{F}\left(\chi^{2}\right)$ that is small and nonempty. This can be achieved better if we work with bounding functions that have similar properties as $F(a)$ and therefore also produce similar search spaces. We therefore make use of Lemma 2, but now with $\lambda$ taken as the smallest and largest eigenvalue of the inverse conditional baseline variance matrix. Our choice of bounding functions is, therefore, given by the following Lemma.

Lemma 3 Let the smallest and largest eigenvalue of the inverse conditional baseline variance matrix $B^{\mathrm{T}} Q_{y y}^{-1} B$ be given as $\lambda_{\min }$ and $\lambda_{\max }$, respectively. Then $F(a)$ is bounded from below and from above by the functions

$$
\begin{aligned}
& F_{1}(a)=\|\hat{a}-a\|_{Q_{\hat{a} \hat{a}}}^{2}+\frac{\lambda_{\min }}{1+\sigma_{l}^{2} \lambda_{\min }}(l-\|\hat{b}(a)\|)^{2} \\
& F_{2}(a)=\|\hat{a}-a\|_{Q_{\hat{a} \hat{a}}}^{2}+\frac{\lambda_{\max }}{1+\sigma_{l}^{2} \lambda_{\max }}(l-\|\hat{b}(a)\|)^{2}
\end{aligned}
$$

Proof Since $\|\hat{b}(a)-b\|_{\frac{1}{\lambda_{\min }} I_{p}}^{2} \leq\|\hat{b}(a)-b\|_{Q_{\hat{b}(a) \hat{b}(a)}}^{2} \leq$ $\|\hat{b}(a)-b\|_{\frac{1}{\lambda_{\max }} I_{p}}^{2}$ for all $\hat{b}(a)$ and for all $b$, it follows that $F_{1}(a) \leq F(a) \leq F_{2}(a)$ for all $a$.

It is our experience, that this chosen set of bounding functions, combined with the above described search and shrink strategy, is a very efficient way for computing the global integer minimizer of our ambiguity objective function $F(a)$. Typical run times are comparable to that of the standard LAMBDA method. The efficiency of the method is also illustrated by the effectiveness of the shrinkage. In our experience, the final number of integer vectors in the reduced search space $\Omega_{1}\left(\chi_{1}^{2}\right), \chi_{1}^{2}=F_{1}\left(\breve{a}_{2}\right)$, is very small and often simply equal to one.

Next to the methods fast numerical performance, it also achieves a very significant increase in success rate when compared to the unconstrained method. This is shown in Table 3 where the single-frequency, single-epoch ILS success rates $\left(\sigma_{l}^{2} \rightarrow \infty\right)$ are compared with the corresponding QC-ILS success rates $\left(\sigma_{l}^{2} \rightarrow 0\right)$, for different measurement precisions and different number of tracked satellites.

\section{Summary and conclusions}

In this contribution, we developed new ILS theory for the GNSS compass. It extends current unconstrained ILS theory to the quadratically constrained case. This extension is
Table 3 Single-frequency, single-epoch, GPS ambiguity success rates for the unconstrained $(U)$ and constrained $(C)$ LAMBDA methods

\begin{tabular}{lllll}
\hline$\sigma_{\phi}[\mathrm{mm}]$ & & \multicolumn{3}{l}{3} \\
\cline { 3 - 4 }$\sigma_{p}[\mathrm{~cm}]$ & & 30 & 15 & 5 \\
\hline \# Sats & Method & \multicolumn{3}{l}{ Success rate $(\%)$} \\
\hline 5 & $\mathrm{U}$ & 3.50 & 20.0 & 86.2 \\
& $\mathrm{C}$ & 73.7 & 86.4 & 99.5 \\
6 & $\mathrm{U}$ & 23.3 & 67.4 & 96.8 \\
7 & $\mathrm{C}$ & 96.6 & 99.6 & 99.9 \\
& $\mathrm{U}$ & 49.9 & 80.4 & 99.4 \\
8 & $\mathrm{C}$ & 99.4 & 99.9 & 100 \\
& $\mathrm{U}$ & 86.0 & 93.9 & 100 \\
& $\mathrm{C}$ & 99.7 & 100 & 100 \\
\hline
\end{tabular}

particularly suited for GNSS compass IAR problems, since for such problems the baseline length may often be assumed known a priori. In our development, we have treated the quadratic constraint in a weighted form, thus allowing the constraint to be either hard (infinite weight), soft (small weight) or absent (zero weight). In this way, our results include the standard unconstrained IAR results as special case.

Although the use of baseline length information for IAR is not new, our model formulation and method of solution are new. Following the quadratically constrained ILS principle, we derived the corresponding ambiguity and baseline estimators. Our ambiguity estimator $\check{a}$ is the integer minimizer of the ambiguity objective function

$F(a)=\|\hat{a}-a\|_{Q_{\hat{a} \hat{a}}}^{2}+\min _{b \in \mathbb{R}^{p}} H(a, b)$

where $H(a, b)=\|\hat{b}(a)-b\|_{Q_{\hat{b}(a) \hat{b}(a)}^{2}}^{2}+\sigma_{l}^{-2}(l-\|b\|)^{2}$. Both type of constraints, the integer ambiguity constraints and the baseline length constraint, are rigorously integrated in the ambiguity objective function. They both contribute to the search and to the success rate.

Once the integer minimizer of $F(a)$ is found, precise directional information (e.g. heading, elevation) can be computed from the fixed baseline estimator. It is given as

$\check{b}=\arg \min _{b \in \mathbb{R}^{p}} H(\check{a}, b)$

The inclusion of the baseline length constraint increases the strength of the GNSS model and in particular enables one to obtain higher ambiguity success rates. The inclusion of the constraint also introduces, however, an additional curvature which results in a more complex ambiguity resolution process, in particular in the case of very short baselines. Since the non-quadratic second term of (49) prohibits the use of standard search methods, a new search strategy was devised. Our search and shrink strategy makes use of bounding functions of $F(a)$ that avoid the usually computational intensive evaluation of $\min _{b \in \mathbb{R}^{p}} H(a, b)$ during the search. We described the search and the shrinking steps of our strategy, including 
the different ways of setting the initial size of the search space. We also indicated the very high success rates that can be achieved with the method. We believe that it will be difficult to devise a method that, with the same information, is capable of achieving higher success rates.

We also presented different special cases and approximations to the integer minimization problem (49). Whether they are practically useful depends on the strength of the unconstrained GNSS model, the length of the baseline and on the precision of the conditional baseline. For the precise determination of GNSS-compass information, we may therefore discriminate between the following four classes of problems:

Class I: If already the unconstrained GNSS model has sufficient strength, one can base the fixed baseline computation on the standard ILS estimator

$$
\check{a}=\arg \min _{a \in \mathbb{Z}^{n}}\|\hat{a}-a\|_{Q_{\hat{a} \hat{a}}}^{2}
$$

In this case, no baseline length constraint is needed $\left(\sigma_{l}^{-2}=0\right)$ and the standard LAMBDA method can be applied. This situation does generally not apply to the single-frequency, singleepoch case (cf. Table 1), but is applicable in the multi-frequency case.

Class II: Since the nonlinearity of $F(a)$ gets smaller for longer baselines, one may use a quadratic approximation of (49) if the baseline is not too short (cf. Table 2). The integer ambiguity vector is then computed as

$$
\check{a}=\arg \min _{a \in \mathbb{Z}^{n}}\|\bar{a}-a\|_{\left(\partial_{a a}^{2} F(\bar{a})\right)^{-1}}^{2}
$$

with $\partial_{a a}^{2} F(\bar{a})$ the Hessian matrix evaluated at the constrained float ambiguity vector $\bar{a}$. As with the problems of Class I, this class permits the application of the standard LAMBDA method. The input is differently though: $\bar{a}$ and $\partial_{a a}^{2} F(\bar{a})$ versus $\hat{a}$ and $Q_{\hat{a} \hat{a}}^{-1}$.

Class III: If the conditional baseline variance matrix is close enough to a scaled unit matrix, one may approximate the constrained ILS ambiguity solution as

$$
\check{a}=\arg \min _{a \in \mathbb{Z}^{n}}\left(\|\hat{a}-a\|_{Q_{\hat{a} \hat{a}}}^{2}+\mu(l-\|\hat{b}(a)\|)^{2}\right)
$$

This approximation has the advantage over (52) that no restrictions are put on the baseline length. Its computation, however, is more complex than the standard LAMBDA method. It is somewhat simpler though, than solving for (54).

Class IV: If the underlying unconstrained GNSS model is not of sufficient strength, or if the baseline length is not long enough, or if the conditional baseline variance matrix is not close enough to a scaled unit matrix, then the above approximate ambiguity solutions will not achieve a large enough success rate. Hence, in that case one will have to compute the constrained ILS ambiguity vector rigorously as

$$
\check{a}=\arg \min _{a \in \mathbb{Z}^{n}}\left(\|\hat{a}-a\|_{Q_{\hat{a} \hat{a}}}^{2}+\min _{b \in \mathbb{R}^{p}} H(a, b)\right)
$$

This solution can be efficiently computed by means of the given search and shrink strategy.

Acknowledgments The research of the author has been supported by an Australian Research Council Federation Fellowship (project number FF0883188). This support is gratefully acknowledged. He also thanks Gabriele Giorgi and Peter Buist for their numerical and creative contributions to the presented theory.

Open Access This article is distributed under the terms of the Creative Commons Attribution Noncommercial License which permits any noncommercial use, distribution, and reproduction in any medium, provided the original author(s) and source are credited.

\section{References}

Chun C, Park FC (1995) Dynamics-based attitude determination using the global positioning system. J Guid Cont Dyn 24(3):466-473

Cohen CE, Parkinson BW (1992) Integer ambiguity resolution of the GPS carrier for spacecraft attitude determination. Adv Astronaut Sci 78(8):91-118

Counselman CC, Gourevitch SA (1981) Miniature interferometer terminals for earth surveying: ambiguity and multipath with the Global Positioning System. IEEE Trans Geosci Remote Sens 19(4):244-252

Crassidis JL, Markley FL, Lightsey EG (1999) Global positioning system integer ambiguity resolution without attitude knowledge. AIAA J Guid Cont Dyn 22(2):212-218

Dai L, Ling KV, Nagarajan N (2004) Real-time attitude determination for microsatellite by LAMBDA method combined with Kalman filtering. In: Proceedings 22nd AIAA international communications satellite systems conference and exhibit 2004 (ICSSC), Monterey, California, USA, p 5

DeLorenzo DS, Alban S, Gautier J, Enge P (2004) GPS attitude determination for a JPALS Testbed: integer initialization and testing. In: Proceedings of position location and navigation symposium PLANS 2004, pp 762-770

de Jonge P, Tiberius C (1996) The LAMBDA method for integer ambiguity estimation: implementation aspects. LGR Series 12 . Publications of the Delft Geodetic Computing Centre, Delft

Furuno (2003) Model SC-120: Satellite compass. http://www. furunocojp/english/indexhtml 
Golub GH, Van Loan CF (1989) Matrix computations, 2nd edn. The Johns Hopkins Univeristy Press, Baltimore

Gomez SF, Lammers ML (2004) Lessons learned from two years of onorbit global positioning system experience on international space station. In: Proceedings of ION-GNSS, Long Beach, US, p 9

Han S, Rizos C (1999) Single-epoch ambiguity resolution for real-time GPS attitude determination with the aid of one-dimensional optical fiber gyro. GPS Solut 3(1):5-12

Hatch R (1990) Instantaneous ambiguity resolution. In: Proceedings of KISć690, Banff, Canada, pp. 299-308

Hauschild A, Montenbruck O (2007) GPS based attitude determination for microsatellites. In: Proceedings of ION GPS 2007, p 11

Hauschild A, Grillmayer G, Montenbruck O, Markgraf M, Vorsmann P (2008) GPS based attitude determination for the flying laptop satellite. Small satellites for earth observation. Springer, Netherlands 211-220

Hayward RC, Gebre-Egziabher D, Powell JD (1998) GPS-based attitude for aircraft. In: Proceedings of 5th Saint Petersburg international conference on integrated navigation systems, pp 85-94

Hofmann-Wellenhof B, Lichtenegger H, Collins J (2001) Global positioning system: theory and practice, 5 th edn. Springer, Berlin

Hofmann-Wellenhof B, Lichtenegger H, Wasle E (2008) GNSS global navigation satellite systems: GPS, GLONASS, Galileo and more. Springer, Berlin

Juang JC, Huang GS (1997) Development of GPS-based attitude determination algorithms. IEEE Trans Aerosp Electr Syst 33(3):968976

Knight D (1994) A new method of instantaneous ambiguity resolution. In: Proceedings of ION-GPS, Colorado Springs, USA, pp 707-716

Kuylen LV, Boon F, Simsky A (2005) Attitude determination methods used in the polarx2@ multi-antenna gps receiver. In: Proceedings of ION GNSS 2005, p 11

Kuylen LV, Nemry P, Boon F, Simsky A, Lorga JFM (2006) Comparison of attitude performance for multi-antenna receivers. Eur J Navig 4(2):1-9

Leick A (2003) GPS satellite surveying, 3rd edn. Wiley, New York

Li Y, Zhang K, Roberts C, Murata M (2004) On-the-fly GPS-based attitude determination using single- and double-differenced carrier phase measurements. GPS Solut 8:93-102

Lin D, Voon LK, Nagarajan N (2004) Real-time attitude determination for microsatellite by LAMBDA method combined with Kalman filtering. In: 22nd AIAA international communication satellite systems conference, Monterey, US, p 8

Mader GL (1990) Ambiguity function techniques for gps phase initialization and kinematic solutions. In: Proceedings of 2nd international symposium on precise positioning with the global positioning system, Ottawa, Canada, pp 1233-1247

Misra P, Enge P (2006) Global positioning system: signals, measurements, and performance. Ganga-Jamuna Press, Lincoln
Monikes R, Wendel J, Trommer GF (2005) A modified LAMBDA method for ambiguity resolution in the presence of position domain constraints. In: Proceedings of ION GPS-2005, pp 81-87

Park C, Kim I (1998) Integer ambiguity resolution for GPS based attitude determination system. In: Proceedings of the 37th SICE annual conference, pp 1115-1120

Park C, Teunissen PJG (2003) A new carrier phase ambiguity estimation for GNSS attitude determination systems. In: Proceedings of international GPS/GNSS symposium, Tokyo, $\mathrm{p} 8$

Park C, Kim I, Lee JG, Jee GI (1996) Efficient ambiguity resolution using constraint equation. In: Proceedings of IEEE: position, location and navigation symposium, PLANSć696, Atlanta, Georgia, USA, pp 227-284

Parkinson B, Spilker JJ (1996) GPS: theory and applications, vols. 1, 2. AIAA, Washington

Povalyaev AA, Sorokina IA, Glukhov PB (2006) Ambiguity resolution under known base vector length. In: Proceedings of ION-ITM GNSS, pp 1413-1417

Remondi BW (1990) Pseudo-kinematic GPS results using the ambiguity function method. NOAA Technical Memorandum NOS NGS52

Strang G, Borre K (1997) Linear algebra, geodesy, and GPS. Wellesley-Cambridge Press, Wellesley

Teunissen PJG (1990) Nonlinear least-squares. Manuscr Geod 15(3): $137-150$

Teunissen PJG (1993) Least squares estimation of the integer GPS ambiguities. Invited lecture, Section IV theory and methodology, IAG General Meeting, Beijing (also in: LGR series No6, Delft Geodetic Computing Center, Delft University of Technology)

Teunissen PJG (1995) The least-squares ambiguity decorrelation adjustment: a method for fast GPS integer ambiguity estimation. J Geod 70:65-82

Teunissen PJG (1998) Success probability of integer GPS ambiguity rounding and bootstrapping. J Geod 72:606-612

Teunissen PJG (1999) An optimality property of the integer leastsquares estimator. J Geod 73(11):587-593

Teunissen PJG, Kleusberg A (1998) GPS for geodesy, 2nd edn. Springer, Berlin

Tu CH, Tu KY, Chang FR, Wang LS (1996) GPS compass: a novel navigation equipment. IEEE Trans Aerosp Electr Syst 33(3):10631068

Wang B, Miao L, Wang S, Shen J (2009) A constrained LAMBDA method for GPS attitude determination. GPS Solut 13:97-107

Wang B, Miao L, Wang S, Shen J (2009b) An integer ambiguity resolution method for the global positioning system (GPS)-based land vehicle attitude determination. Meas Sci Technol 20:11

Wang Y, Zhan X, Zhang Y (2007) Improved ambiguity function method based on analytical resolution for GPS attitude determination. Meas Sci Technol 18:2985-2990 